\title{
Khawa Karpo: Tibetan Traditional Knowledge and Biodiversity Conservation
}

Jan Salick and Robert K. Moseley. 2012. Missouri Botanical Garden, St. Louis. Pp. 273. US\$55 (paperback). ISBN 978-1-935641-06-3

\author{
Reviewed by Denise M. Glover
}

Reviewer address: Department of Sociology and Anthropology, University of Puget Sound, Tacoma, WA, USA. dglover@pugetsound.edu

It is for good reason that Jan Salick will be receiving the Distinguished Economic Botanist award (awarded by the Society for Economic Botany) this month at the joint Society of Ethnobiology and Society for Economic Botany conference in Cherokee, NC. The work by Salick and Robert Moseley, which benefits from additional collaborating authors in several chapters and appendices, is impressive, especially in its breadth. This publication demonstrates the significant contributions that Salick and others have made to our understanding of the ecology and biodiversity, as well as local knowledge of this, in the greater Khawa Karpo area in China's Southwest, or the southern reaches of cultural Tibet.

The overall strengths of the work include establishing a baseline for conservation agendas, as little research has been done on this topic in this area of the world and even a smaller amount is published in English; this the authors recognize as a main goal of the publication. Additionally valuable are the appendices, which contain important information on flora and fauna in the region; the compilation of a comprehensive list of vascular plants in the region is especially inclusive. Also essential is the discussion of local Tibetan knowledge, particularly although not exclusively from doctors of Tibetan medicine, in the context of conservation. The authors urge that such local knowledge, in addition to local ideas of sacred sites, needs to be recognized as the important resource that it is for conservation efforts. To ethnobiologists, this is hardly news, but unfortunately to some conservation biologists this point needs repeating - and who better to bring this home than Salick and her team? The time-depth of the research (spanning close to a decade) is very important as well and, although not highlighted in the book, is another demonstration of the significant commitment to conservation and dialogue about conservation that Salick and her team have. Lastly, the value of this work in terms of documenting climate change and local responses to it cannot be under-estimated.

Some of the innovative aspects of research that has been done in this region by Salick and colleagues and which are discussed in the book include the use of photographs, both as a form of documentary analysis about climate change (comparing current photos to photos taken of the same area 100 years ago to see changes in the landscape over time) and as a form of ethnographic data aimed at highlighting the emic perspective on related issues (this is a technique used by anthropologists since the early days of selfreflexive ethnographic film, but has over the past decade or so become more widely used in other fields).

As a Tibetologist but also an ethnobiologist from the anthropological lineage that works in the same region (as well as on related topics), the weaknesses of the book for me mainly revolve around shortfalls in understanding (or simplifications of) Tibetan language, culture, and the contemporary state of Tibetan medicine. Somewhat ironically (given the interest in dialogue with Tibetan communities), there are few Tibetan names for plants and animals given. While translation between different taxonomic systems can be challenging, they are not impossible and seem woefully lacking in this monograph. There are some errors as well: confusion of Wade-Giles and Wylie systems of transliteration (Sman ri is in fact Wylie, not Wade Giles); the translation of sman as "medicinal 
herb" is somewhat misleading, since sman in Tibetan refers to many types of materia medica (not just botanicals); the elision of "fraternal" to describe the kind of polyandry practiced in this area. Other problems include the repeated use of pinyin romanization for Tibetan names (I am very aware of the difficulties of this in SW China especially but effort must be made to avoid this); the fact that the authors appear to have used Indian sources (and not Tibetan ones) to discuss the history of Tibetan medicine (use of the name Vairotsana for the historic figure that Tibetans call Yuthog Yonten Gonpo suggests this); and several statements about the state of Tibetan medicine in the People's Republic of China seem simplistic and outdated: saying that traditional Tibetan medicine is recognized by the government only if from Lhasa does not acknowledge the contemporary central role of Tibetan medicine practice, teaching, research, and production in the Amdo (Qinghai) region and in Chamdo, two main hubs of activity in Tibetan medicine. There is some conflation of Bön and Buddhist concepts of the sacredness of land, and the characterization of Bön as being about good and evil is simplistic. The last issue I have with the monograph is that it does not directly cite sources (although acknowledged in a bibliography); this is likely my bias as an academic, and clearly the audience that the book hopes to reach is not restricted to academics alone.

Nonetheless, regardless of these few shortcomings, the book is well worth the read and is a significant contribution to the field. The mix of accessiblywritten text and beautiful color photos makes this a valuable asset not only to scholars of conservation studies, ethnobiology, and Tibetan studies, but also to anyone interested in learning more about this very special part of the world, the area surrounding the impressive and sacred Khawa Karpo Mountain. 